\title{
Direct and Indirect Effect of Different Yield Contributing Traits in Indian Mustard (Brassica juncea L. Czern \& Coss)
}

\author{
Kuldeep Yadav $^{1 *}$, Mahak Singh ${ }^{1}$, Rajendra Kumar Yadav', \\ Abhinav Yadav ${ }^{2}$, Soni Singh ${ }^{3}$ and Durga Prasad ${ }^{1}$ \\ ${ }^{1}$ Department of Genetics and Plant Breeding, Chandra Shekhar Azad University of \\ Agriculture and Technology, Kanpur, (U.P.) India \\ ${ }^{2}$ CSIR - National Botanical Research Institute, Rana Pratap Marg, Lucknow, India \\ ${ }^{3}$ Department of Genetics and Plant Breeding, Narendra Deva University of Agriculture and \\ Technology, Kumarganj, Ayodhya, (U.P.) India \\ *Corresponding author
}

\section{A B S T R A C T}

\section{Keywords}

Path analysis, Direct effect, indirect effect and Indian mustard

\section{Article Info}

Accepted:

22 January 2021

Available Online:

10 February 2021
The present investigation consisting a set in a fashion of half diallel cross combinations were carried out to investigate the inter characters relationship for fifteen characters in Indian mustard (Brassica juncea L. Czern \& Coss) at oilseed research farm, Kalyanpur of Chandra Shekhar Azad University of Agriculture \& Technology, Kanpur under during rabi, 2018-19. The observations were recorded for days to 50\% flowering, days to maturity, plant height $(\mathrm{cm})$, length of main raceme $(\mathrm{cm})$, leaf area index, number of primary branches per plant, number of secondary branches per plant, number of siliquae per plant, number of seeds per siliqua, biological yield per plant (g), 1000-seeds weight $(\mathrm{g})$, harvest index $(\%)$, oil content $(\%)$, protein content $(\%)$ and yield per plant $(\mathrm{g})$. In this study, Trait harvest index has highest direct effect on seed yield followed by some other traits at both genotypic and phenotypic path analysis. Resultant direct selection for these traits would be effective for further yield improvement in given genotype of Indian mustard.

\section{Introduction}

Indian mustard (Brassica juncea L. Czern \& Coss) is an important Rabi season oilseed crop; belongs to family Cruciferae and genus Brassica. Indian mustard is a natural amphidiploid $(2 \mathrm{n}=36)$ of Brassica rapa $(2 \mathrm{n}=$ 20) and Brassica nigra $(2 \mathrm{n}=16)$. Mustard is largely self-pollinated but certain amount (2 $15 \%$ ) of cross pollination may take place due to honeybees. Mustard seed is the world's second leading source of vegetable oil, after soybean. It is also the second most leading source of protein meal in the world after soybean. It is mainly grown in northern part of India; Rajasthan is the largest producing state followed by M.P \& Uttar Pradesh. Mustard crop required lower water requirement (240$400 \mathrm{~mm}$ ) for completing life cycle, therefore it is fits well for rain fed cropping system. Mustard seed contains average $34-43 \%$ oil content and contributes for $32 \%$ of total edible 
oil. India is the third largest rapeseed-mustard producer in the world after China and Canada with 12 percent of world's total production (20016-17). Among the four oleiferous Brassica species, major area is under Brassica juncea, which contributes about 80 per cent of the total rapeseed-mustard production in the country. Among the various oilseed crops grown globally, the estimated area, production and yield of rapeseed-mustard in the world was $36.68 \mathrm{mha}, 72.42 \mathrm{mt}$ and1974 $\mathrm{kg} / \mathrm{ha}$, respectively, during 2017-18. Globally India account for $19.80 \%$ and $9.8 \%$ of the total acreage and production. In India estimated area production and productivity of rapeseed \& mustard was $6.07 \mathrm{mha}, 7.92 \mathrm{mt}$ and $1304 \mathrm{~kg}$ /ha (Anon. 2017-18). In Uttar Pradesh estimated area, production and Yield was 0.66 mha, $0.84 \mathrm{mt}$ and $1080 \mathrm{~kg} / \mathrm{ha}$, respectively (Anon. 2016-17). Yield is complex character which dependent on the various yield contributing characters. Thus, the study of correlation between yield and its component is of primary importance in formulating the selection criteria under crop improvement. Selection of any desirable trait is generally performed based on the phenotypic value of the plants, which is partly determined by genotypes, which is heritable, and partly by environment which is non- heritable. Therefore, it is necessary to know the various components of the yield and its mutual correlation with other independent traits.

Path coefficient analysis is a partial regression technique which separates the direct effects from the indirect effects through other related characters by partitioning the correlation coefficient into direct and indirect effects on yield. It is most efficient method to establish relationships among the variables that affect grain yield as it allows determination of the relative magnitude of each. Therefore, the objective of this study was to assess the association among yield and yield contributing traits and identify traits those have the most direct and indirect effects on grain yield.

\section{Materials and Methods}

The basic experimental material comprised of 10 genotypes of Indian mustard (Brassica juncea) namely, Varuna, Rohini, Vardan, PR20, PR-21, Kranti, Narendra Ageti Rai-7, Narendra Rai-8501, KMR 17-3 and KMR 174 were selected on the basis of morphological diversity for different agronomical traits and their 45 hybrids for investigation. The observations were recorded on various quantitative characters viz. days to $50 \%$ flowering, days to maturity, plant height $(\mathrm{cm})$, length of main raceme $(\mathrm{cm})$, leaf area index, number of primary branches per plant, number of secondary branches per plant, number of siliquae per plant, number of seeds per siliqua, biological yield per plant (g) 1000 -seeds weight $(\mathrm{g})$, harvest index $(\%)$, oil content $(\%)$, protein content $(\%)$ and yield per plant $(\mathrm{g})$. In this study, ten plants in random way were selected in each row of each replication for study. All the characters were recorded under study except days to $50 \%$ flowering and days to maturity which were recorded on plot basis. Path coefficient was worked out as method suggested by Dewey and Lu (1959).

\section{Results and Discussion}

A character contributing to seed yield may contribute directly or indirectly. The estimates of direct and indirect effect are presented in (Table 1a). At phenotypic level, harvest-index (0.8250) displayed high order of direct positive effect on seed yield per plant followed by biological yield per plant (0.5578), oil content (0.0085), number of secondary branches per plant (0.0071), days to maturity (0.0036), 1000-seed weight (0.0029) and number of seeds per siliqua (0.0013). 
Table.1(a) Phenotypic path coefficients parents $+\mathrm{F}_{1}$ for 15 characters in 10 x 10 diallel cross in Indian mustard (Brassica juncea L.)

\begin{tabular}{|c|c|c|c|c|c|c|c|c|c|c|c|c|c|c|c|}
\hline Characters & $\begin{array}{l}\text { Days to } \\
50 \% \\
\text { flowering }\end{array}$ & $\begin{array}{l}\text { Days to } \\
\text { maturity }\end{array}$ & $\begin{array}{l}\text { Plant } \\
\text { height } \\
\text { (cm) }\end{array}$ & $\begin{array}{l}\text { Length of } \\
\text { main } \\
\text { raceme }\end{array}$ & $\begin{array}{l}\text { Leaf } \\
\text { area } \\
\text { index }\end{array}$ & $\begin{array}{l}\text { No. of } \\
\text { primary } \\
\text { branches } \\
\text { /plant }\end{array}$ & $\begin{array}{l}\text { No. of } \\
\text { secondary } \\
\text { branches/pl } \\
\text { ant }\end{array}$ & $\begin{array}{l}\text { No. of } \\
\text { siliquae / } \\
\text { plant }\end{array}$ & $\begin{array}{l}\text { No. of } \\
\text { seeds / } \\
\text { siliqua }\end{array}$ & $\begin{array}{l}\text { Biologica } \\
\text { l yield / } \\
\text { plant (g) }\end{array}$ & $\begin{array}{l}1000- \\
\text { seeds } \\
\text { weight } \\
\text { (g) }\end{array}$ & $\begin{array}{l}\text { Harvest } \\
\text { index } \\
(\%)\end{array}$ & $\begin{array}{l}\text { Oil } \\
\text { content } \\
(\%)\end{array}$ & $\begin{array}{l}\text { Protein } \\
(\%)\end{array}$ & $\begin{array}{l}\text { Seed } \\
\text { yield / } \\
\text { plant }\end{array}$ \\
\hline $\begin{array}{l}\text { Days to } 50 \% \\
\text { flowering }\end{array}$ & -0.00760 & 0.00240 & -0.00290 & -0.00010 & -0.00120 & 0.00210 & 0.00220 & -0.00340 & -0.00020 & 0.24320 & 0.00120 & 0.01080 & 0.00200 & 0.00000 & $0.248 * *$ \\
\hline Days to maturity & -0.00520 & 0.00360 & -0.00520 & -0.00020 & -0.00170 & 0.00030 & 0.00320 & -0.00450 & 0.00000 & 0.37530 & 0.00150 & 0.13040 & 0.00270 & 0.00000 & $0.500 * *$ \\
\hline Plant height (cm) & -0.00330 & 0.00280 & -0.00660 & -0.00010 & -0.00140 & 0.00030 & 0.00190 & -0.00390 & 0.00010 & 0.38210 & 0.00150 & 0.07220 & 0.00160 & 0.00000 & $0.447 * *$ \\
\hline $\begin{array}{l}\text { Length of main } \\
\text { raceme }(\mathrm{cm})\end{array}$ & -0.00260 & 0.00190 & -0.00270 & -0.00030 & -0.00190 & -0.00160 & 0.00450 & -0.00500 & 0.00020 & 0.31770 & 0.00120 & 0.31510 & 0.00590 & -0.00010 & 0.632 ** \\
\hline Leaf area index & -0.00320 & 0.00220 & -0.00330 & -0.00020 & -0.00280 & -0.00280 & 0.00500 & -0.00630 & 0.00030 & 0.33990 & 0.00230 & 0.54920 & 0.00680 & 0.00000 & $0.887 * *$ \\
\hline $\begin{array}{l}\text { Number of } \\
\text { primary } \\
\text { branches/plant }\end{array}$ & 0.00150 & -0.00010 & 0.00020 & 0.00000 & -0.00070 & -0.01070 & 0.00140 & -0.00150 & 0.00010 & 0.03930 & 0.00070 & 0.29760 & 0.00250 & 0.00000 & $0.330 * *$ \\
\hline $\begin{array}{l}\text { Number of } \\
\text { secondary } \\
\text { branches/plant }\end{array}$ & -0.00230 & 0.00160 & -0.00180 & -0.00020 & -0.00200 & -0.00210 & 0.00710 & -0.00540 & 0.00030 & 0.26110 & 0.00140 & 0.42420 & 0.00530 & 0.00000 & $0.687 * *$ \\
\hline $\begin{array}{l}\text { Number of } \\
\text { siliquae/plant }\end{array}$ & -0.00350 & 0.00220 & -0.00340 & -0.00020 & -0.00230 & -0.00220 & 0.00510 & -0.00750 & 0.00040 & 0.32400 & 0.00190 & 0.44570 & 0.00610 & 0.00000 & $0.766^{* *}$ \\
\hline $\begin{array}{l}\text { Number of } \\
\text { seeds/siliqua }\end{array}$ & 0.00100 & 0.00000 & -0.00060 & 0.00000 & -0.00060 & -0.00100 & 0.00150 & -0.00240 & 0.00130 & 0.05540 & 0.00020 & 0.24590 & 0.00290 & 0.00000 & $0.304 * *$ \\
\hline $\begin{array}{l}\text { Biological } \\
\text { yield/plant (g) }\end{array}$ & -0.00330 & 0.00240 & -0.00450 & -0.00020 & -0.00170 & -0.00080 & 0.00330 & -0.00430 & 0.00010 & 0.55780 & 0.00150 & 0.00800 & 0.00350 & 0.00000 & $0.562 * *$ \\
\hline $\begin{array}{l}\text { 1000-seed weight } \\
\text { (g) }\end{array}$ & -0.00300 & 0.00190 & -0.00330 & -0.00010 & -0.00220 & -0.00270 & 0.00350 & -0.00480 & 0.00010 & 0.28700 & 0.00290 & 0.52120 & 0.00560 & 0.00000 & $0.806^{* * *}$ \\
\hline Harvest index (\%) & -0.00010 & 0.00060 & -0.00060 & -0.00010 & -0.00190 & -0.00390 & 0.00360 & -0.00400 & 0.00040 & 0.00540 & 0.00180 & 0.82500 & 0.00570 & 0.00000 & $0.832 * *$ \\
\hline Oil content $(\%)$ & -0.00180 & 0.00110 & -0.00130 & -0.00020 & -0.00220 & -0.00320 & 0.00440 & -0.00540 & 0.00050 & 0.22840 & 0.00190 & 0.55320 & 0.00850 & 0.00000 & $0.784 * *$ \\
\hline Protein $(\%)$ & 0.00120 & -0.00050 & 0.00010 & 0.00010 & 0.00010 & 0.00000 & -0.00080 & 0.00100 & 0.00020 & -0.03250 & 0.00040 & 0.05900 & 0.00040 & 0.00020 & 0.029 \\
\hline
\end{tabular}

Residual effect: 0.0330 
Table.1(b) Genotypic path coefficients parents $+\mathrm{F}_{1}$ for 15 characters in 10 x 10 diallel cross in Indian mustard (Brassica juncea L.)

\begin{tabular}{|c|c|c|c|c|c|c|c|c|c|c|c|c|c|c|c|}
\hline Characters & $\begin{array}{c}\text { Days to } \\
\mathbf{5 0 \%} \\
\text { flowering }\end{array}$ & $\begin{array}{l}\text { Days to } \\
\text { maturity }\end{array}$ & $\begin{array}{c}\text { Plant } \\
\text { height } \\
\text { (cm) }\end{array}$ & $\begin{array}{l}\text { Length of } \\
\text { main } \\
\text { raceme }\end{array}$ & $\begin{array}{l}\text { Leaf } \\
\text { area } \\
\text { index }\end{array}$ & $\begin{array}{c}\text { No. of } \\
\text { primary } \\
\text { branches } \\
\text { /plant }\end{array}$ & $\begin{array}{c}\text { No. of } \\
\text { secondary } \\
\text { branches/p } \\
\text { lant }\end{array}$ & $\begin{array}{c}\text { No. of } \\
\text { siliquae / } \\
\text { plant }\end{array}$ & $\begin{array}{l}\text { No. of } \\
\text { seeds / } \\
\text { siliqua }\end{array}$ & $\begin{array}{l}\text { Biologica } \\
1 \text { yield / } \\
\text { plant (g) }\end{array}$ & $\begin{array}{c}1000- \\
\text { seeds } \\
\text { weight } \\
\text { (g) }\end{array}$ & $\begin{array}{c}\text { Harvest } \\
\text { index } \\
(\%)\end{array}$ & $\begin{array}{c}\text { Oil } \\
\text { content } \\
(\%)\end{array}$ & $\begin{array}{c}\text { Protein } \\
(\%)\end{array}$ & $\begin{array}{l}\text { Seed } \\
\text { yield / } \\
\text { plant }\end{array}$ \\
\hline $\begin{array}{l}\text { Days to } 50 \% \\
\text { flowering }\end{array}$ & 0.011 & 0.106 & -0.070 & 0.031 & -0.102 & 0.005 & 0.006 & -0.020 & -0.027 & 0.279 & 0.067 & 0.010 & -0.017 & -0.001 & $0.280 * *$ \\
\hline Days to maturity & 0.008 & 0.149 & -0.123 & 0.048 & -0.136 & 0.000 & 0.009 & -0.025 & -0.004 & 0.434 & 0.084 & 0.137 & -0.023 & 0.000 & $0.556^{* *}$ \\
\hline Plant height (cm) & 0.005 & 0.127 & -0.145 & 0.034 & -0.101 & 0.001 & 0.005 & -0.020 & 0.017 & 0.406 & 0.077 & 0.077 & -0.013 & 0.000 & $0.469 * *$ \\
\hline $\begin{array}{l}\text { Length of main } \\
\text { raceme }(\mathrm{cm})\end{array}$ & 0.004 & 0.088 & -0.061 & 0.081 & -0.146 & -0.003 & 0.011 & -0.026 & 0.022 & 0.347 & 0.065 & 0.336 & -0.047 & -0.001 & $0.670 * *$ \\
\hline Leaf area index & 0.006 & 0.102 & -0.074 & 0.059 & -0.198 & -0.006 & 0.013 & -0.034 & 0.039 & 0.366 & 0.125 & 0.598 & -0.054 & 0.000 & $0.943 * *$ \\
\hline $\begin{array}{l}\text { Number of } \\
\text { primary } \\
\text { branches/plant }\end{array}$ & -0.004 & -0.002 & 0.008 & 0.018 & -0.088 & -0.014 & 0.005 & -0.011 & 0.032 & 0.045 & 0.059 & 0.493 & -0.030 & 0.000 & $0.510 * *$ \\
\hline $\begin{array}{l}\text { Number of } \\
\text { secondary } \\
\text { branches/plant }\end{array}$ & 0.005 & 0.098 & -0.051 & 0.066 & -0.191 & -0.005 & 0.013 & -0.036 & 0.039 & 0.333 & 0.096 & 0.556 & -0.052 & 0.000 & $0.870 * *$ \\
\hline $\begin{array}{l}\text { Number of } \\
\text { siliquae/plant }\end{array}$ & 0.005 & 0.097 & -0.076 & 0.055 & -0.172 & -0.004 & 0.012 & -0.039 & 0.051 & 0.341 & 0.098 & 0.481 & -0.048 & 0.000 & $0.802 * *$ \\
\hline $\begin{array}{l}\text { Number of } \\
\text { seeds/siliqua }\end{array}$ & -0.003 & -0.005 & -0.022 & 0.016 & -0.069 & -0.004 & 0.005 & -0.017 & 0.113 & 0.072 & 0.011 & 0.345 & -0.032 & 0.001 & $0.411 * *$ \\
\hline $\begin{array}{l}\text { Biological } \\
\text { yield/plant (g) }\end{array}$ & 0.006 & 0.118 & -0.108 & 0.051 & -0.133 & -0.001 & 0.008 & -0.024 & 0.015 & 0.544 & 0.084 & 0.097 & -0.029 & 0.000 & $0.628 * *$ \\
\hline $\begin{array}{l}\text { 1000-seed weight } \\
\text { (g) }\end{array}$ & 0.005 & 0.083 & -0.073 & 0.035 & -0.163 & -0.005 & 0.008 & -0.025 & 0.008 & 0.301 & 0.151 & 0.556 & -0.043 & 0.000 & $0.836 * *$ \\
\hline $\begin{array}{l}\text { Harvest index } \\
(\%)\end{array}$ & 0.000 & 0.025 & -0.014 & 0.034 & -0.148 & -0.009 & 0.009 & -0.023 & 0.049 & 0.066 & 0.105 & 0.802 & -0.048 & 0.000 & $0.848 * *$ \\
\hline Oil content (\%) & 0.003 & 0.051 & -0.028 & 0.058 & -0.163 & -0.006 & 0.011 & -0.028 & 0.054 & 0.239 & 0.099 & 0.590 & -0.066 & 0.000 & $0.814 * *$ \\
\hline Protein (\%) & -0.002 & -0.023 & 0.001 & -0.026 & 0.005 & 0.000 & -0.002 & 0.005 & 0.022 & -0.034 & 0.020 & 0.058 & -0.003 & 0.003 & 0.025 \\
\hline
\end{tabular}

Residual effect: 0.0164 
The highest negative direct effect was exerted by number of primary branches per plant (0.0107), days to $50 \%$ flowering ($0.0076)$,number of siliquae per plant $(-0.0075)$ and plant height (-0.0066).Biological yield per plant exhibited high order of positive indirect effects on seed yield per plant via plant height (0.3821) followed by days to maturity (0.3753), leaf area index (0.3399), number of siliquae per plant $(0.3240)$ and length of main raceme (0.3177); harvest index via oil content (0.5532) followed by leaf area index (0.5492), 1000-seed weight (0.5212), number of siliquae per plant (0.4457), number of secondary branches per plant (0.4242) and length of main raceme (0.3151).

At genotypic level (Table 1b), close examination of the result revealed that harvestindex (0.802) displayed high order of direct positive effect on seed yield per plant followed by biological yield per plant (0.544),1000-seed weight (0.151), days to maturity (0.149) and number of seeds per siliqua (0.113).

The highest negative direct effect was exerted by leaf area index (-0198) followed by plant height (-0.145), oil content (-0.066) and number of siliquae per plant $(-0.039)$.

Biological yield per plant contributed to seed yield per plant via days to maturity (0.434), followed by plant height (0.406), leaf area index (0.366), length of main raceme (0.347), number of siliquae per plant (0.341) and number of secondary branches per plant (0.333); harvest index via leaf area index (0.598) followed by oil content (0.590), number of secondary branches per plant (0.556), 1000-seed weight (0.556) and number of primary branches per plant (0.493); days to maturity contributed moderate indirect effect to seed yield per plant via plant height $(0.127)$ followed by biological yield per plant (0.118) and days to $50 \%$ flowering (0.106);1000-seed weight via leaf area index $(0.125)$ followed by harvest index $(0.105)$ and oil content (0.099); number of seeds per siliqua contributed low indirect effect to seed yield per plant via oil content (0.054) followed by number of siliquae per plant (0.051) and harvest index (0.049); length of main raceme via number of secondary branches per plant (0.066), leaf area index (0.059) and oil content (0.058).

In conclusion the rest of the estimates of indirect effects obtained in phenotypic and path analysis were negligible. In present study residual effect for both levels was occurred with value 0.0164 at genotypic and 0.0330 at phenotypic level indicating that some other factors which were not been considered in the study, must be included in the analysis to explain total variation in seed yield.

The study of character association and path coefficient analysis indicated that leaf area index and harvest index had positive direct effect coupled with positive significant correlation with grain yield per plant and hence direct selection can be made based on this trait for improving grain yield in Indian mustard.

\section{References}

Anonymous, Area, production, productivity of mustard in India and Karnataka. 2016-17. www.indiastat.com.

Anonymous, Canola history, 2017-18. www.canolacouncil. org.

Deway DI, Lu KH. A correlation and pathcoefficient analysis of components of crested wheatgrass seed production. Agron. J. 1959; 51:515-518.

\section{How to cite this article:}

Kuldeep Yadav, Mahak Singh, Rajendra Kumar Yadav, Abhinav Yadav, Soni Singh and Durga Prasad. 2021. Direct and Indirect Effect of Different Yield Contributing Traits in Indian Mustard (Brassica juncea L. Czern \& Coss). Int.J.Curr.Microbiol.App.Sci. 10(02): 3107-3111. doi: https://doi.org/10.20546/ijcmas.2021.1002.339 